\title{
Design and Fabrication of Small Vertical-Take- Off-Landing Unmanned Aerial Vehicle
}

\author{
Yasir Ashraf Abd Rahman ${ }^{1}$, Mohammad Taghi Hajibeigy ${ }^{1,}$, Abdulkareem Shafiq Mahdi \\ Al-Obaidi ${ }^{1}$, and Kean How Cheah $^{2}$ \\ ${ }^{1}$ School of Engineering, Taylor's University, Malaysia \\ ${ }^{2}$ School of Engineering and Physical Sciences, Heriot-Watt University, Malaysia
}

\begin{abstract}
Modern UAVs available in the market have well-developed to cater to the countless field of application. UAVs have their own limitations in terms of flight range and manoeuvrability. The traditional fixed-wing UAVs can fly for long distance but require runways or wide-open spaces for take-off and landing. On the other hand, the more trending multirotor UAVs are extremely manoeuvrable but cannot be used for long-distance flights because of their slower speeds and relatively higher consumption of energy. This study proposed the implementation of hybrid VTOL UAV which has the manoeuvring advantage of a multirotor UAV while having the ability to travel fast to reach a further distance. The design methodology and fabrication method are discussed extensively which would be followed by a number of flight tests to prove the concept. The proposed UAV would be equipped with quadcopter motors and a horizontal thrust motor for vertical and horizontal flight modes respectively.
\end{abstract}

Keywords: UAV, VTOL, RC aircraft, drone

\section{Introduction}

Unmanned Aerial Vehicles (UAV) are aircrafts which are controlled remotely via direct radio frequency communication or are equipped with autonomous devices to performed autonomous mission flights. The active development in the field of UAV in recent years has led to its increase in versatility in terms of its functionality and range of application. Early UAVs development is generally divided into two separate types, Fixed Wing UAV (FWUAV), and rotorcraft UAV (RUAV). FWUAVs typically have longer endurance and thus can reach further than RUAV in a single flight, but require runways for take-offs and landings. RUAVs on the other hand, are more manoeuvrable and able to take-off and land vertically without any runway but has much lesser endurance due to their low efficiency in power consumption and low speed.

\footnotetext{
${ }^{1}$ Corresponding author: MohammadTaghi.Hajibeigy@,Taylors.edu.my
} 
Therefore, to bridge the performance gap between RUAVs and FWUAVs, the solution is to have a hybrid Vertical-Take-Off-Landing (VTOL) aircraft. This type of aircraft will have the advantages of both FWUAV and RUAV and to a substantial extent should be able to eliminate their shortcomings. In the aviation industry, major companies have already successfully mass-produced and commercialized numerous models of VTOL aircraft. A famous example of such aircraft is the Bell Boeing V-22 Osprey which combined the features of fixed wing aircraft and rotary wing aircraft via the concept of tilt rotor mechanism. The tilt-rotor mechanism is only one of the concepts that were proven for successful VTOL flights. Other researchers have also explored other concepts to incorporate into similar hybrid VTOL aircrafts. In the small to medium UAV applications, particularly in the Radio-Controlled (RC) drone world, only a few small-scale projects have been successful in flying VTOL aircrafts [1]. Also, those projects that are successful experienced great difficulty in optimizing the transition flight from the rotor to fixed-wing flight and vice versa. In addition, manufacturers are still developing their own VTOL UAV for aerial-photography drone market, but they are still yet to be commercialized. 
The hybrid VTOL UAV design, however, has its own shortcomings compared to both FWUAV and RUAV. The addition of extra propulsion system to cater for horizontal and vertical flights resulted in significant extra weight carried by the vehicle. Normally, a conventional RUAV model would only need four brushless motors for its vertical propulsion and other manoeuvres. An addition of horizontal flight would require at least an extra motor for horizontal propulsion as well as a number of servo motors for different manoeuvres. There is also a notable increase in the complexity of the design and control mechanism. The common VTOL UAV design can be classified into three types. This include 1) TYPE1, which is a fixed wing aircraft with tilting rotor mechanism, 2) TYPE2, which has no wings and has fixed vertical rotor(s), 3) TYPE3, which has wings and vertical rotor(s) [2].

Each of these designs has its own complexity, with the TYPE1 being most complex than others. Moreover, due to the difficulty in optimizing the transition flight from vertical to horizontal flight and vice versa, the VTOL UAV may lose its balance during the transition process and can lose a lot of altitudes. Therefore, it is vital to design the VTOL UAV that is lightweight, able to perform transition flight safely and also relatively simple yet efficient propulsion system.

This study reviews previous attempts at developing a VTOL UAVs as well as current projects and combine suitable concepts into a better efficient prototype of a VTOL UAV. Particularly, this study would emphasize the control development on the transition flight mode for better stability and safety. This study proposed the implementation of a TYPE3 VTOL UAV without the tilting mechanism as the scope of this research. TYPE3 VTOL UAV is the least complex system and better stability and safety during the transition flight mode as compared to the VTOL UAV with the tilting mechanism. Another factor of the choice of VTOL type is limited time and cost factor.

The proposed aircraft motor configuration would consist of four motors for its vertical propulsion, a single motor for the horizontal propulsion and servo motors on the control surface for horizontal flight manoeuvring. Basically, the vertical propulsion motors would be attached to the fixed wing platform to produce a hybrid VTOL UAV system. Unlike the tilt-rotor VTOL UAV, the vertical propulsion motors would not tilt and would function to fulfil the vertical flight mode while the horizontal motor is used for horizontal flight. This would reduce the system's complexity as a result of different flight modes are controlled by separate actuators.

In this paper, theoretical concepts behind the flight dynamics of a VTOL UAV are established which would be the basis of this study. Later, the detailed design process is presented where the estimations, calculations, selection of materials, conceptual designs and 3D modellings are justified by the mission requirement stated. At the end of the design process, fabrication and assembly process of a VTOL UAV prototype are done which are followed by a series of software simulations and actual flight tests to support the concept of a non-tilting rotor VTOL UAV.

The concepts of how a fixed-wing aircraft and multirotor works are quite simple. However, developing a VTOL hybrid aircraft requires integrating the flight characteristics of both fixed-wing aircraft and multirotor platforms. Therefore, it is important to first have a separate understanding of the general theory of how a fixed-wing aircraft and multirotor aircraft works as well as the effective control technique to stabilize the whole system.

The primary aim of this project is to determine the potential performance of a small VTOL UAV. First, the VTOL UAV is to be designed and fabricated using scientific methodologies. Then, a number of flight tests are performed under different weather conditions to assess the stability performance during hover, transition and winged flight. The secondary aim is to identify the flight duration of a VTOL UAV compared to a common RUAV of similar specifications.

\section{Theoretical Backgrounds 2.1 General Theory}

Basically, a VTOL aircraft has the ability to fly like a fixed-wing aeroplane and at the same time hover like a multirotor. A body-fixed coordinate system is usually used in flight dynamics where the coordinate system is fixed relative to the aircraft.

For a multirotor aircraft in motion, it is appropriate to classify the three rotation axes that can alter the craft's attitude as 1) Roll: Rotation of x-axis, 2) Pitch: Rotation of y-axis, 3) Yaw, rotation of z-axis. These axes of rotation are shown in Figure 1 below. 


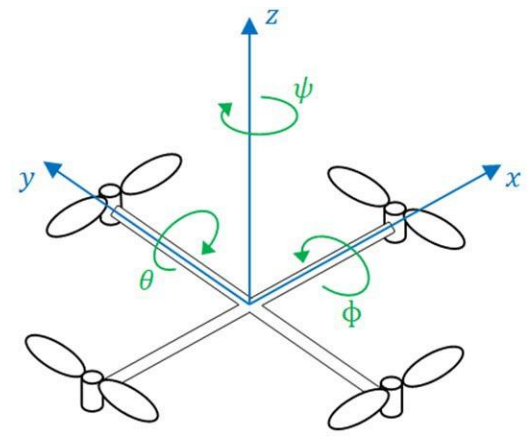

Fig. 1. The coordinate system of a multirotor aircraft.

A common multirotor propeller configuration usually consists of four propellers or better known as a quadcopter. Other known multirotor configurations include tricopter, hexacopter and octocopter. Different configurations are for the different area of application and have performance variation in terms of flight time, agility, stability and payload capacity [3][4]. The propeller's main task is to generate enough lift force to bring the aircraft up to the sky and also deliver adequate thrust to move its current position to forward, backward, left and right. The attitude of multirotors is actuated by the difference in rotation speed of each propeller. Figure 2 shows how the aircraft's attitude change and the corresponding body movement when the rotation speed of certain propellers is manipulated [5]. There is four basic movements for multirotors.

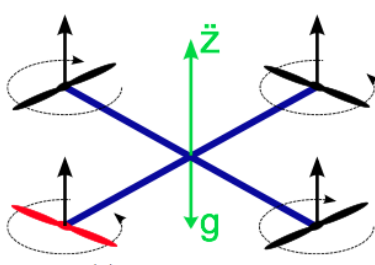

(a) Upward movement

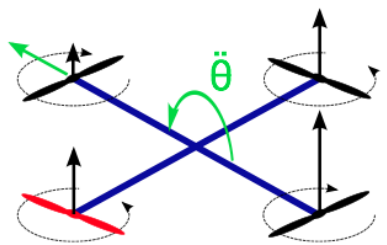

(c) Roll and lateral movement

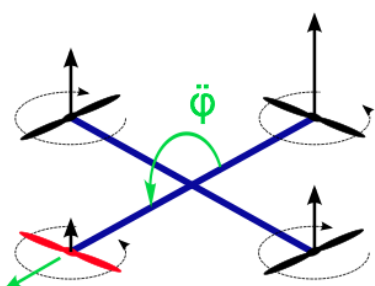

(b) Pitch and lateral movement

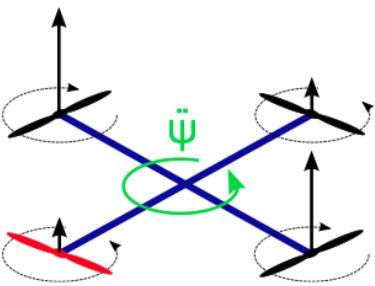

(d) Yaw and rotational movement

Fig. 2. Attitude and Body Movement Change due to rotation speed change in the individual propeller.

The multirotor must be able to achieve a reasonably stable flight during hover before attempting to manoeuvre it in its pitch, roll and yaw axes. Stable flight requires that the pitch and roll axes must be first stabilized. The multirotor will be at risk of tipping over and crash if these axes are not controlled adequately. The pitch and roll attitudes are determined using the gyroscope and accelerometer which is built onboard the Flight Controller Unit (FCU). As for the yaw axis, it is less critical to stabilizing the axis provided that the multirotor is still controllable. Unstable yaw axis would slightly drift the multirotor body in that axis but can be balanced by using the radio controller.

For a fixed-wing aeroplane in motion, the coordinate system is similar to that of the multirotor. A clear difference is that aeroplanes use propellants that are aligned to its fuselage for forwarding propulsion as depicted in Figure 3 below. Lift in this case is generated by the aerodynamic pressure difference between the upper and lower surface of the wing. The attitude of the aircraft is controlled using the control surfaces attached to the main wing, horizontal tail wing and vertical tail wing. The three control surfaces are called 1) Elevator: Control the pitch axis, 2) Aileron: Control the roll axis, 3) Rudder: Control the yaw axis. 


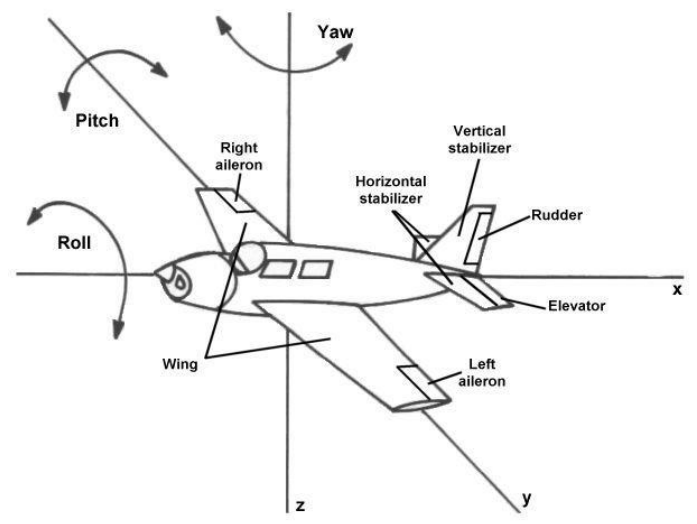

Fig. 3. Coordinate System of a fixed-wing aircraft [6].

Similar to multirotors, to fly fairly stable while in straight-and-level flight, the roll and pitch axes must be stabilized properly. In full-scale manned aircraft, this can be done by directly trimming the ailerons and elevators. In the case of small-scale unmanned fixed-wing aircraft, the roll and pitch axes can be stabilized by using the attitude sensors such as the accelerometer and gyroscope.

Designing and fabricating a hybrid VTOL UAV system involves considering the flight dynamics of both multirotor and fixed-wing systems. The dynamics would become more complex due to propulsion units for longitudinal and vertical movements. To simplify the hybrid VTOL UAV concept, during vertical flight mode, the UAV is assumed to follow the flight dynamics of a multirotor system, whereas, during horizontal flight mode, it is expected that the craft's flight dynamics follow fixed-wing flight dynamics. During the transition flight mode, the aircraft will be in a varying stated and the gravity can be overcome by both vertical and longitudinal propulsion units until the craft has complete the transition process. Optimizing the altitude requires the controlling motor outputs properly. In the transition to horizontal flight mode, lack of vertical motor outputs while not achieving minimum stall speed would severely decrease the altitude. Inversely, while transiting to vertical flight mode, reducing the longitudinal motor output below minimum stall speed before the vertical motors achieve adequate thrust would lead to reducing altitude. It should be noted that maximum airspeed is limited to the rated power of the longitudinal propulsion unit. Moreover, additional structure to support the vertical propulsion system may add to the drag during horizontal flight mode. However, these extra drags can be counteracted by having wings with high lift coefficient characteristics.

\subsection{PID Control Technique}

Proportional, integral and derivative (PID) control is one of the most attractive techniques used to stabilize as well as optimize the control of UAVs [7]. During the flight, it is preferred to have a stable flight where the aircraft maintained its desired attitude effortlessly without external inputs from the user. In cases where wind or other external disturbance detected, the FCU as processing unit will compute the errors between current and desired attitude and perform necessary corrections. PID control tries to balance out this disturbance by giving out more motor and/or servo outputs accordingly. Figure 4 represents a general block diagram for any PID control system.

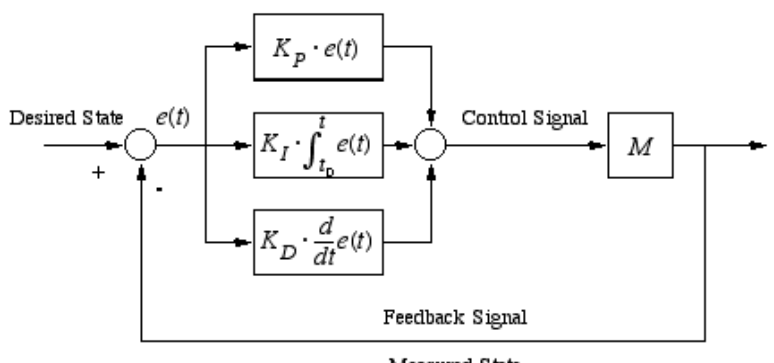

Measured State

Fig. 4. General PID Block Diagram. 
PID control can be easily understood by representing it in the block diagram shown in Figure 4 above. The steps for PID control are:

1. The desired state is first determined and the previously measured state is presented at the beginning of the loop to compute the state error $e(t)=$ Desired State - Measured State

2. The proportional, integral and derivative terms are calculated as $K_{P} * e(t), K_{I}^{*}$ (integral of $\left.e(t)\right)$ and $K_{D} *($ derivative of $e(t))$ respectively.

3. The 3 terms are totaled up to obtain the motor/servo output, $u(t)=P+I+D$

In reality, it would be difficult to obtain a stable system by concurrently tuning the control gains, $\left(K_{P}, K_{I}\right.$ and $\left.\mathrm{K}_{\mathrm{D}}\right)$ of the PID controller on all three axes. Therefore, the more critical axes, which are the pitch and roll are first stabilized separately before the yaw axis. PID Control method will yield the desired stability performance characteristics on all axes when the control gains are tuned correctly.

PID control is the most widely used method in theoretical approach and engineering applications partly because exhaustive dynamics models are not required to implement this method. Obtaining the best control gain parameters can be done by modifying the gains live during flight tests according to the control effect of the UAV until the desired response is achieved [8]. Many researchers who have resorted to developing UAV based on PID control have achieved stable flights on their experimental platforms in different modes. Therefore, due to its reliability, PID control is adopted in this study.

\section{Design Methodology}

\subsection{Flow Chart}

The flow chart for the overall research design shown in Figure 5 was prepared at the beginning of this project. This flow chart will serve as the general guidance to steer this project in the right direction. The flowchart is divided into two parts, in which the first part concentrate on the design and the second part focus on the fabrication and flight tests. The outcome of this research is to study the performance of the proposed hybrid VTOL UAV by analysis of flight data recorded during test flights.

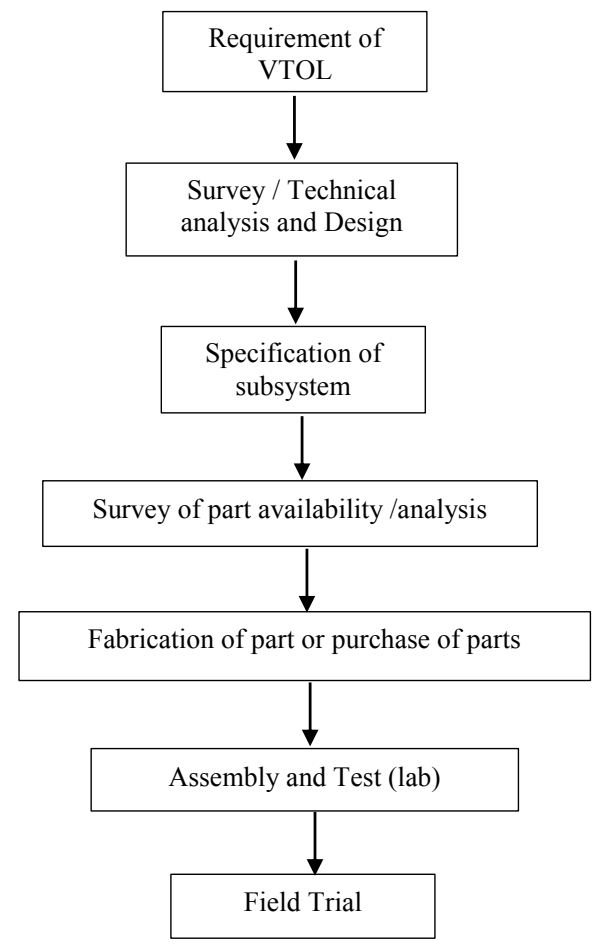

Fig. 5. Design Methodology Flowchart 


\subsection{Specifying Requirements}

The requirements of the proposed TYPE3 VTOL UAV are established after considering the information obtained from a literature survey conducted. These requirements are set in a way that the UAV is practically feasible in term of design, fabrication and flight test.

There are a few basic UAV requirements established in this study. First, TYPE3 VTOL UAV, which consists of conventional fixed wing aircraft attached with fixed vertical rotors to be incorporated into the VTOL design. The UAV should have long flight range and ability to reach the desired location as fast as possible at the reasonable time. The UAV should be manoeuvrable at narrow and confined spaces like a helicopter. Maximum payload of $1 \mathrm{~kg}$ is to be allowed which may consist of imaging equipment, gimbals, etc. The UAV should be capable of performing VTOL operations with acceptable safety and stability characteristics. The transition flight mode from rotor flight to fixed-wing flight and vice versa is to be handled manually or autonomously. All-Up-Weight (AUW) of less than $3.5 \mathrm{~kg}$. The wingspan of about $1.5-2.0 \mathrm{~m}$. Five-rotor system (vertical quadrotors and single horizontal rotor) for stability and reduction in complexity. The expected cruise velocity range of approximately $15-20 \mathrm{~m} / \mathrm{s}$. The stall speed is thus should be around $70 \%$ of the minimum cruise velocity. Lastly, it would be equipped with versatile flight controller unit able to handle manual controls and execute autonomous missions. The final build of this proposed prototype is roughly shown in Figure 6 where in the future, minor modifications may be necessary.

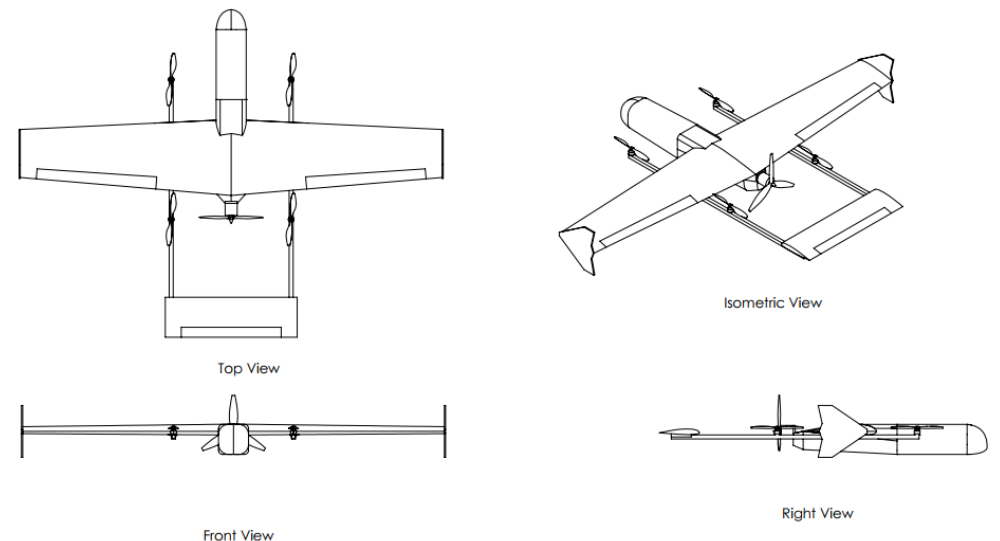

Fig. 6. Overview of the proposed VTOL UAV.

\subsection{Test Protocol}

The prototype would be conducted in both manual and autonomous flights. The UAV would take off vertically in vertical flying mode, then perform transition to horizontal flying mode, fly straight and level to a certain designated waypoint, then perform transition to vertical flying mode again and finally land the UAV safely. For a manual flight, a ground user would pilot the UAV via radio control transmission. This mission profile can be simply visualized as in Figure 7 below.

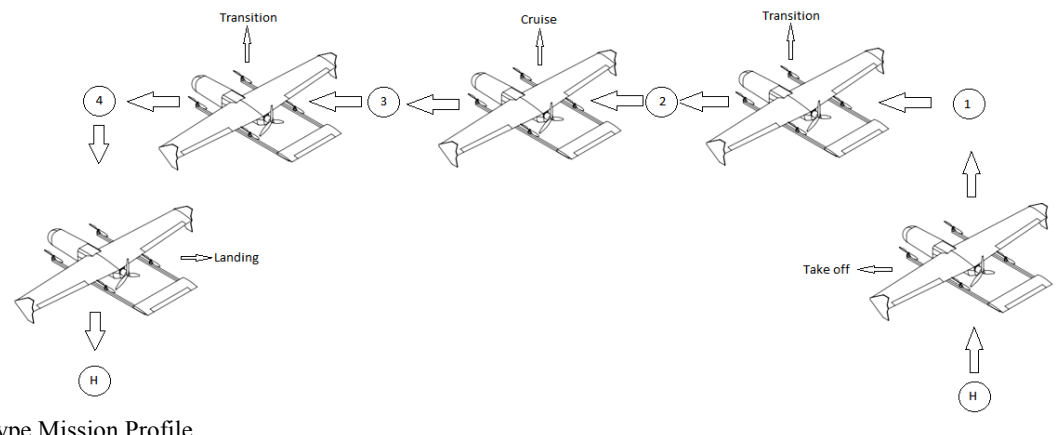

Fig. 7. Prototype Mission Profile. 
From Figure 7, the biggest challenge is to perform the transition flight from horizontal flight to vertical flight mode and vice versa. During this portion of the flight, the UAV may lose some altitude due to the reduction in lift force vector while slowing down the vertical motors. Hence, the horizontal motors need to produce enough thrust to accelerate the UAV quickly for the wings to replace the vertical motors in generating lift. Another method to address this possible altitude lose is to develop an effective control system where the vertical motors would continue to generate enough lift while in transition until it passed the minimum stall speed.

\subsection{Structural Design Parameters}

To design a small-scale VTOL UAV, it is important to limit certain parameters of sizes to a practically reasonable figure so that other parameters concerning dimensions would be feasible for fabrication. The design parameters chosen should also obey known theoretical concepts and equations to confirm that the VTOL UAV will be able to accomplish stable and safety flights across all its flight modes. The general procedures to determine basic structural design parameters are:

1. Estimate the All-Up-Weight of the aircraft

2. Wing Design: Determine the size of the wing loading, wingspan, aspect ratio and chord

3. Fuselage Design: Determine the size of the fuselage

4. Tail Design: Determine the size of the tail wing and its distance from the main wing

These general procedures will be used to estimate the size of the prototype. After fabrication and flight tests, the actual dimensions will be adjusted accordingly.

\subsection{Weight Estimation}

Before fabricating the prototype, some components which are readily available was weighed while the weight of components that needed to be work is estimated. The total All-Up-Weight (AUW) would be designed slightly higher than the total estimated weights. This is because normally, the actual prototype weight will be higher than the initial weight calculations. Estimating the extra weight also increase the margin for the extra payload. It is important to determine the right AUW for the prototype as the subsequence design calculations are based entirely on this parameter see Table 1.

Table 1. Weight Estimate for proposed prototype

\begin{tabular}{|l|r|r|r|}
\hline Item & Mass (g) & Quantity & Total Mass (g) \\
\hline Motor & 85 & 5 & 425 \\
\hline ESC and PDB & 65 & 5 & 325 \\
\hline Battery 3s 6000mAh & 422 & 1 & 422 \\
\hline FCU, gps, power module, receiver & 114 & 1 & 114 \\
\hline Propeller & 12 & 5 & 60 \\
\hline Servo & 60 & 4 & 240 \\
\hline Aluminium frame & 700 & 1 & 700 \\
\hline Polystyrene Board & 600 & 1 & 600 \\
\hline Plywood base & 200 & 1 & 200 \\
\hline Consumables (bolts, nuts, cable ties, glues, etc) & 150 & 1 & 150 \\
\hline \multicolumn{1}{|c|}{ Total All-Up-Weight } & & & 3236 \\
\hline
\end{tabular}

Hence, AUW would be estimated a bit higher than the calculated value to be $3.5 \mathrm{~kg}$.

\subsection{Wing Design}

First, Wing Loading, $\mathrm{W}_{1}$ is to be calculated (Equation 1).

$$
W_{l}=\frac{A U W}{S_{W}}
$$

The wing loading needs to be as low as possible to ensure minimum stress exerted during flight can be sustained by the wing. Wing loading value is more important than the actual weight of the aircraft because it tells how much weight is 
carried by each unit of wing area, $S_{\mathrm{W}}$. From literature survey, $10 \mathrm{~kg} / \mathrm{m} 2$ is selected which would make $S_{\mathrm{W}}$ be about 0.32 $\mathrm{m}^{2}$. Next, the wingspan, $\mathrm{b}$ can be calculated from the wing's Aspect Ratio formula given in Equation (2) below.

$$
A R=b^{2} / S
$$

Generally, for normal aircrafts that are designed for low-speed flights have typical Aspect Ratios around 5-10. Higher Aspect Ratio increases the gliding capability of the aircraft and can fly at lower speed. AR $=10$ is chosen so that the proposed VTOL UAV can safely glide in case of motor failure. Therefore, $b_{W}=1.8 \mathrm{~m}$.

to determine the wing chord, $\mathrm{C}_{\mathrm{W}}$, the wing is considered to have a rectangular shape, this is shown in Equation 3.

Hence, $\mathrm{C}_{\mathrm{W}}=0.18 \mathrm{~m}$

$$
S=b * C
$$

For the Aileron sizing, the suitable Aileron area, $\mathrm{S}_{\mathrm{a}}=20 \%$ of $\mathrm{S}_{\mathrm{W}}$

Therefore,

$\mathrm{S}_{\mathrm{a}}=0.32 * 0.2=0.064 \mathrm{~m}^{2}$

$\mathrm{b}_{\mathrm{a}}=(0.064 * 10)^{0.5}=0.8 \mathrm{~m}$

$\mathrm{C}_{\mathrm{a}}=0.064 / 0.8=0.08 \mathrm{~m}$

For the chosen wing size, Reynold's number, Re has to be confirmed to be in the laminar region. Reynold's number is given as in Equation 4.

$$
R e=\frac{V C}{v}
$$

At STP air condition, $\rho=1.225 \mathrm{~kg} / \mathrm{m}^{3}, v=1.46 \mathrm{e}-5 \mathrm{~m}^{2} / \mathrm{s}$

For the estimated cruise speeds,

$\operatorname{Re}_{@ V=15 \mathrm{~m} / \mathrm{s}}=1.85 \mathrm{e}-5$

$\mathrm{Re}_{@ \mathrm{~V}=20 \mathrm{~m} / \mathrm{s}}=2.46 \mathrm{e}-5$

Therefore, the flow will be in the laminar region.

\subsection{Tail Design}

For the tail sizing, the calculations are similar to the wing sizing calculations. For this study, a flat horizontal tail consisting of only elevator control surface is chosen. The yaw axis of the prototype would not be controlled as it would add to the complexity and for the time being, is unnecessary to proof the concept of hybrid VTOL UAV. Instead, the yaw axis can still be controlled while the UAV is in vertical flight mode.

The initial step is to determine the location of the tail wing from the main wing. This location should produce enough moment to control the pitch axis. As a general rule, the distance from leading edge of the wing to the leading edge of the tail should be around thrice of $C_{W}$, in this case, it would be approximately $0.6 \mathrm{~m}$.

The next step is to determine the surface area of the horizontal tail, $\mathrm{S}_{\mathrm{ht}}$, which is normally about $30 \%$ of $\mathrm{S}_{\mathrm{W}}$.

$\mathrm{S}_{\mathrm{ht}}=0.3 * 0.32=0.096 \mathrm{~m}^{2}$

The Aspect Ratio of the horizontal tail is taken as 3. The subsequent calculations to find tail span and tail chord are the same as Equation (2) and (3) respectively. This gives $b_{h t}=0.53 \mathrm{~m}$ and $C_{h t}=0.18 \mathrm{~m}$.

For the Elevator sizing, the the suitable Elevator area, $\mathrm{S}_{\mathrm{e}}=20 \%$ of $\mathrm{S}_{\mathrm{ht}}$

Therefore,

$\mathrm{S}_{\mathrm{e}}=0.096 * 0.2=0.0192 \mathrm{~m}^{2}$

$\mathrm{b}_{\mathrm{e}}=(0.0192 * 3) 0.5=0.24 \mathrm{~m}$

$\mathrm{C}_{\mathrm{e}}=0.0192 / 0.24=0.08 \mathrm{~m}$

\subsection{Fuselage Design}

The fuselage does not have any specific calculations as the prototype will use the F450 quadcopter frame (Figure 8) as the base fuselage. This frame's aerodynamic shape can be improved to cater for the horizontal flight mode by covering 
the centre hub using light materials and incorporating shape that can improve lift and reduce drag. Covering the centre hub also protects the exposed main electronics part such as the flight controller unit, battery, GPS unit and power distribution board.

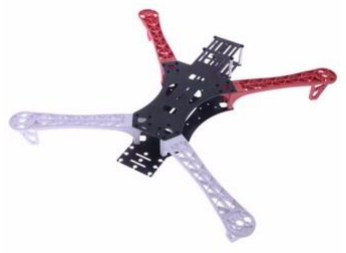

Fig. 8. F450 frame.

\subsection{Material Selection}

Key and critical parameters in the design of such UAV is choice of material. Every single part of the smallest item such as a screw to the largest part such as a wing, weight is the critical factor when coming to selection. Ideally, the lightest material and yet roust against mechanical (drop, shock) and environment (waterproof, salt spray compliant, altitude /low pressure, oil/chemical contamination/corrosion) shall be selected. Such UAV that normally uses in harsh environment shall comply with some international standard of complaint to ensure the product is robust and can be used in any kind of application/situation. High investment in design/fabrication and qualification test shall be anticipated.

However, the final choice of material and choice of manufacturing/fabrication techniques is also depending on cost affordability and quantity to be produced. For this study which is considered a prototype or PoF (Proof of Concept) model, the choice of material is not at all compliant with the above Mil Standard. What most important in this study is the VTOL is really working and demonstrable. Material for the wing should be light enough and easily available. In this study, wing material is made out of Depron foam which is very lightweight, easy to shape and low cost [9]. The frame support would consist of a combination of the ready-made F450 frame to house the electronics components and hollow 1 " by $1 / 2$ " aluminium bar.

\subsection{Electronics Design}

No specific electronic design take place in this stud. Most electronic parts such as the motor, control module, radio frequency modules and battery/charger are COTFs (commercial on the shelf) item. Main activities in the electronics are on parts/systems integration activities.

Key and critical electronic part/subsystem of the UAV are:

- $\quad$ Radio Remote control

- Brushless Motor

- Battery capacity /voltage

\subsubsection{Brushless Motor}

Most important in the selection of this motor are a specification of RPM vs supply voltage and durability and also some spec of waterproof and temperature. Long flight duration could cause heat issue and to some extent, it could melt the insulation of the motor coils and causing a short circuit. It is advisable not to use the motor up to the maximum limit of the voltage supply. For real design for commercial use, waterproof or splash proof is a must for this motor. The IP65 standard which outlined protection against contact and infiltration of water and dirt (IP) standard recognized internationally and is available free download from the internet. For this study which is proof of concept project, a requirement such as IP65 is not really considered. What most important is the capability of the term are RPM and weight.

\subsubsection{Battery}

There are many battery technologies available commercially, named NiCd, Li-Ion, Li-Polymer, Lead Acid etc. For this mobile application, capacity density (how energy stored in a specific volume/weight) is critical. The smaller the size with the higher energy density is the preferred technology. As far as today is concern Li-Ion battery is the most common battery technology for the mobile application. It is a stable technology. Li-polymer is better in term of energy 
capacity and with a flexible shape but is more expensive. NiCd is obsolete technology and Lead Acid is for automotive application.

Rechargeable Li-Polymer battery is the best choice, cheap and good energy capacity and current rating value. In addition, this batter has a good life cycle (charge/discharge) 1000 cycle, long lasting usage.

\subsubsection{Electronics Layout and Operation}

Figure 9 below is the wiring diagram on the electric part of the VTOL-UAV. The heart or the core engine of the system is the Pihawx Flight Control Unit, a control processor unit. Its control the rotation or RPM of the motor during takeoff, landing or horizontal movement of the VTOL-UAV.

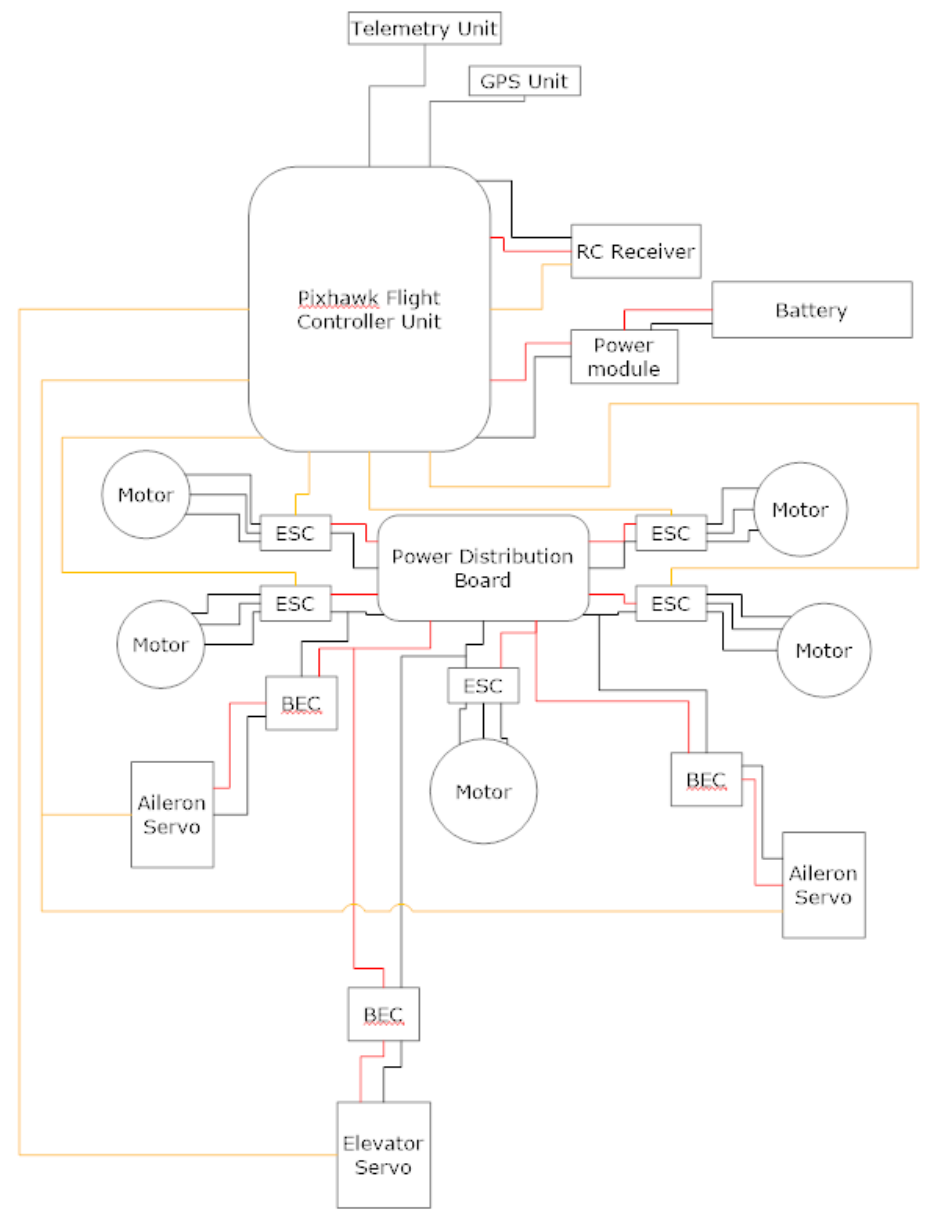

Fig. 9. Wiring Diagram for Onboard.

The input of speed (increase or decrease the motor RPM) come from the operator at the ground. Data was transmitted from the transmitter $\mathrm{RC}$ at the ground to receive remote control on the VTOL-UAV by microwave signal operating at $2.4 \mathrm{Ghz}$ (ISM unlicensed band for commercial application). Other data transmitter from the ground is the controlling /navigation info for the aircraft. The range (how far the UAV can fly from the operator) is determined by the transmitted power of the $\mathrm{RC}$ at the ground and the receiver sensitivity of the RC receiver on board the UAV. It must be a line of sight link, i.e. any blockage such as rain, cloud, trees will degrade the reachable range of the remote control.

Beyond the reachable range of the RC or due to blockage it will cause the VTOL-UAV be flying with control and the risk it falls/ drops to ground will be unavoidable. 
On board of the VTOL-UAV there is GPS and telemetry unit. GPS device read the position, speed? and altitude of the UAV. These GPS data together with information on battery status and sensor (such as a camera) could be sent back to the ground centre the telemetry transmitter operating at $915 \mathrm{Mhz}$. In this prototype, mode camera was not installed as it is not part of the scope of the project.

The supply for all the system comes from the reachable 11.1V LI-Ion battery. The battery could be later changed to $14.8 \mathrm{~V}$ (will extra cell) to increase the RPM of the motor, in case the $11.1 \mathrm{~V}$ does not adequate to lift the VTOL-UAV.

The duration of the flight depends on the capacity of the battery. The higher battery capacity the longer the VTOLUAV can fly.

\subsection{VTOL mechanism}

A key element to ensure the VTOL capability of the four (4) motors with rotary blade stability to lift the load. All the four rotary blades will be at the same speed (Rotation per minute) and acceleration rate i.e. changing the RPM from one state to another state. Increasing the RPM means increasing the height or takeoff stage. Decreasing the speed or RPM mean reducing the altitude or to land. The same RPM for all the vertical blade and weight balance are the keys to ensure stability during takeoff landing and horizontal movement. The speed of the propeller and stability will be achieved by means of PID stability control techniques as described in the earlier section 3.2 above.

Horizontal movement (speed of the UAV) is by mean of controlling the RPM of then horizontal motor/propeller. Attitude control and stability are achieved by adjusting the control surface accordingly and tuning the PID control gains. Manual adjustments of speed/RPM of all motor/propeller are remotely done by mean of radio remote control while during autopilot missions, the UAV would follow the designated speed as programmed in the software.

\subsection{D Modelling}

The 3D modelling is done in Solidworks software. Basically, the critical parts that need to be modelled are the main wing and the horizontal tail wing which would be made from Depron foam. Other parts such as the plastic and aluminium frames are less important to model because these parts are known for their reliability and strength during actual flight test and would not be easily be damaged by light crashes. The 3D modelling for wing section and tail section are shown in Figure 10 and Figure 11 respectively. The steps for the 3D modelling are:

1. Determine the airfoil shape of the wing

2. Project the airfoil cross-section into 3D model

3. Assemble in the wings with the frame

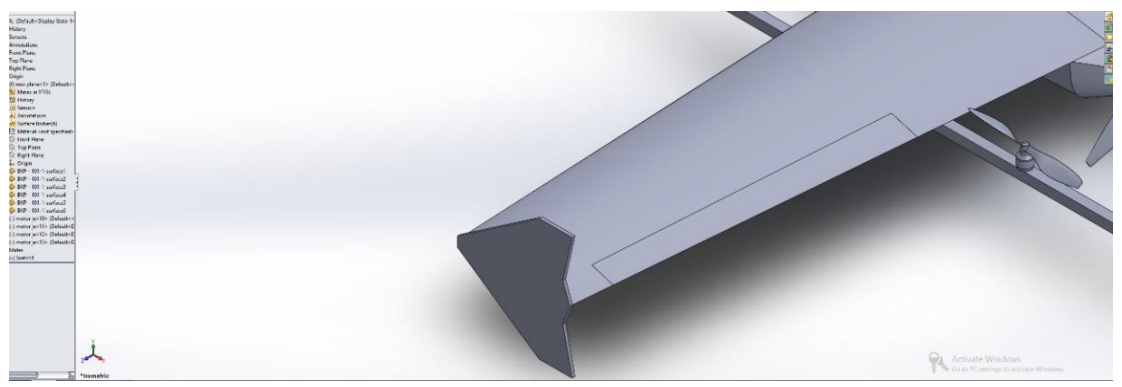

Fig. 10. 3D Modelling of Wing Section.

The cross-section (airfoil shape) of the wing is modelled using standard NACA coordinates which are available online. For the proposed prototype, the suitable airfoil would be the NACA 3414 airfoil family. It has low camber and the lower surface is quite flat and therefore would produce good Lift-Drag ratio for the estimated airspeeds. 


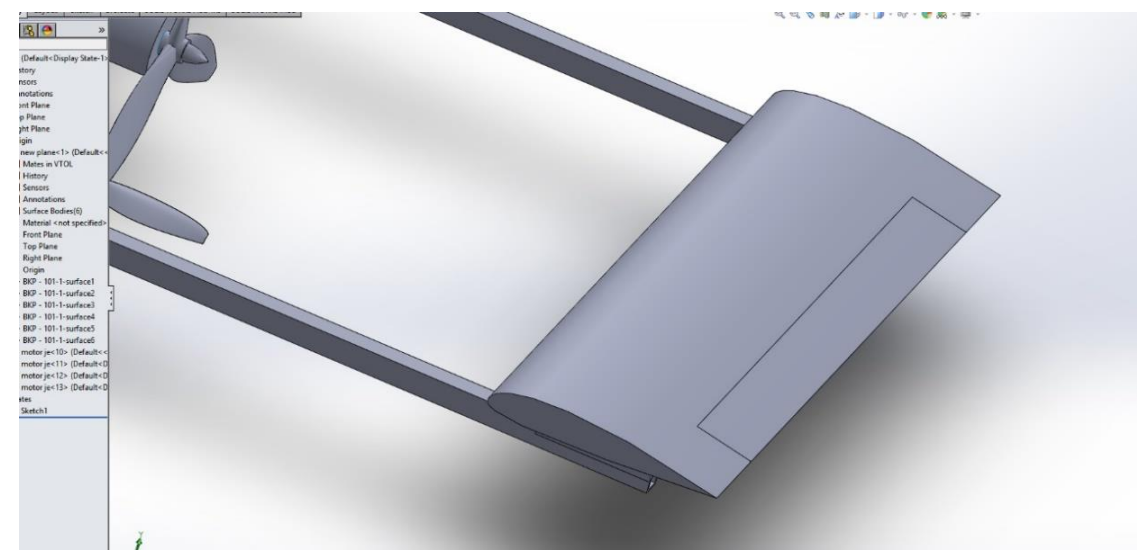

Fig. 11. 3D Modelling of Tail Section.

For the horizontal tail cross-section, NACA 0012 airfoil family is used which is thinner and result in lower drag. As calculated before, the area of the horizontal tail is quite small and therefore the tail is expected to be able to withstand pressure during flight. In addition, both the main and tail wing would be reinforced with aluminium angled beam if the actual flight tests fail to sustain it from crashes.

\subsection{Software setups}

Mission planner software is used to setup the Pixhawk flight controller unit. This software uploads the correct firmware to Pixhawk to match the prototype's vehicle type which is a hybrid VTOL UAV. This software is also used to tune the PID control gains to achieve optimum stability. This can be done during actual flight tests where the gains are adjusted accordingly by the pilot or can be set before flight tests and Trial-and-Error method is used to obtain the best result. Figure 12 shows a screenshot of tuning tab inside Mission Planner.

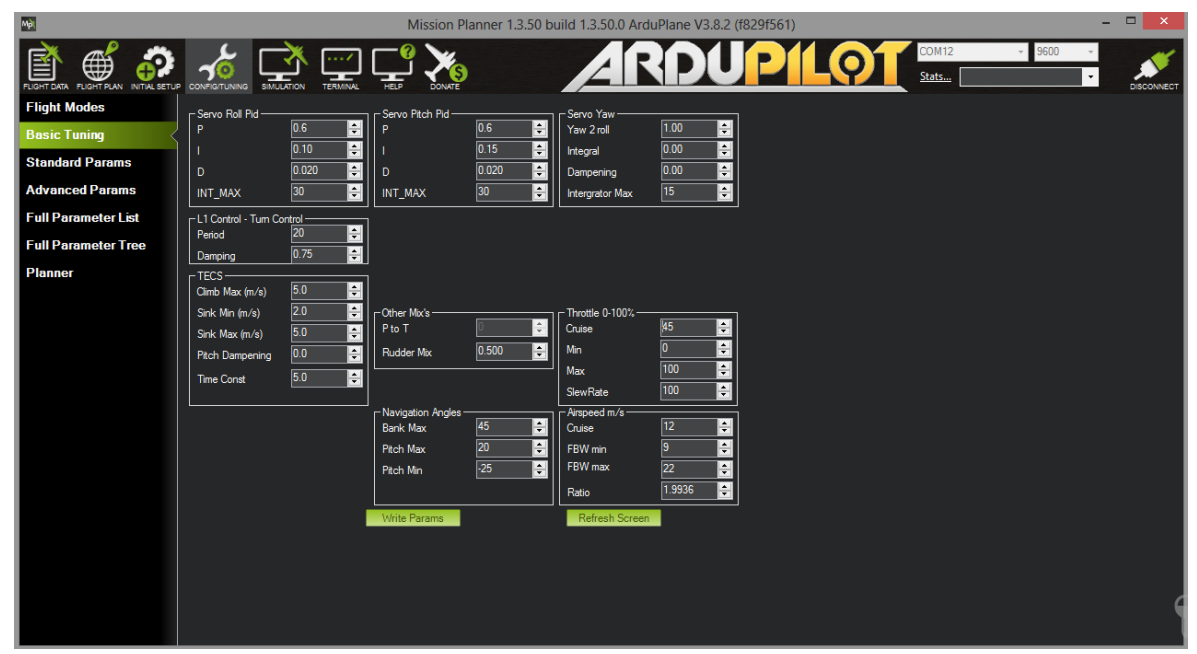

Fig. 12. Mission Planner PID Tuning Tab.

\section{Results and Discussions}

The fabrication was done successfully and the final prototype was tested to be fully functional. The built of the prototype is shown in Figure 13. After complete fabrication and assembly have been done, physical inspection along with subsystems and full system tests were performed. 


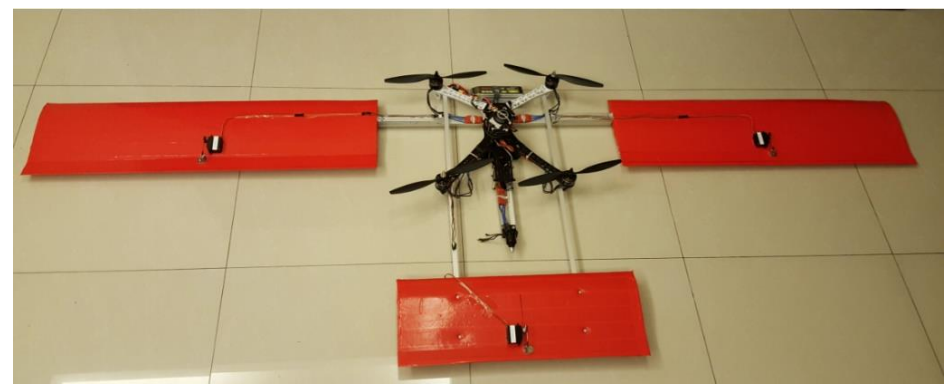

Fig. 13. Final Prototype After Complete Fabrication and Assembly.

Apart from this, the data flash logs from actual flight tests were successfully recorded for further analysis. Using the advanced FCU which is equipped onboard the system, various type of data can be recorded. However, the data that are of interest for this project includes the PID response performance within the 3 axes of rotation, the vibration level about the three axes and also the flight trajectory data.

The flight modes that were used when the data was recorded are stabilized, altitude hold and loiter mode. These three modes are the flight modes in vertical flights. As for the horizontal flight modes, the flight tests could not be done yet due to limitations in available open space that are safe for flight tests. In stabilize mode, the position and altitude control is performed manually. In altitude hold mode, the altitude is controlled autonomously while the UAV position can still be drifted due to the wind. In loiter mode, both altitude and position hold is controlled autonomously. In all these three modes, the accelerometer onboard the FCU is automatically stabilized where minimum user intervention is required to hold the UAV in a perfect hover.

The graph is shown in Figure 14 and Figure 15 highlight the vibration level $(\mathrm{m} / \mathrm{s} / \mathrm{s})$ as well as the roll response $(\mathrm{m} / \mathrm{s} / \mathrm{s})$ against time. This graph displays the flight time of about 1 minute to ease the visualization of the graphs. In summary, the stability performance in a roll and other axes was at acceptable levels where the response of the system, recorded in the unit of $\mathrm{m} / \mathrm{s} / \mathrm{s}$, followed closely to the desired inputs either from the user or the FCU itself as it tried to auto-stabilize the axes continuously. Further tuning of the PID gain values may be required to obtain a more desired performance. As for the vibration levels, the values recorded also fell within an acceptable level of vibrations in all axes (below $60 \mathrm{~m} / \mathrm{s} / \mathrm{s}$ ). This suggests that for future works, it would be suitable to install a camera onboard for in-flight monitoring and for other purposes.

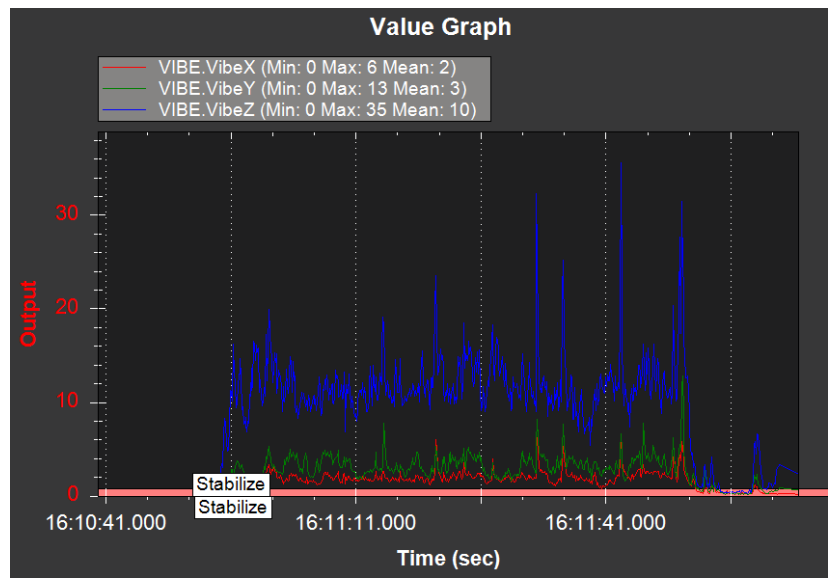

Fig. 14. Graph of Vibration levels in all three axes. 


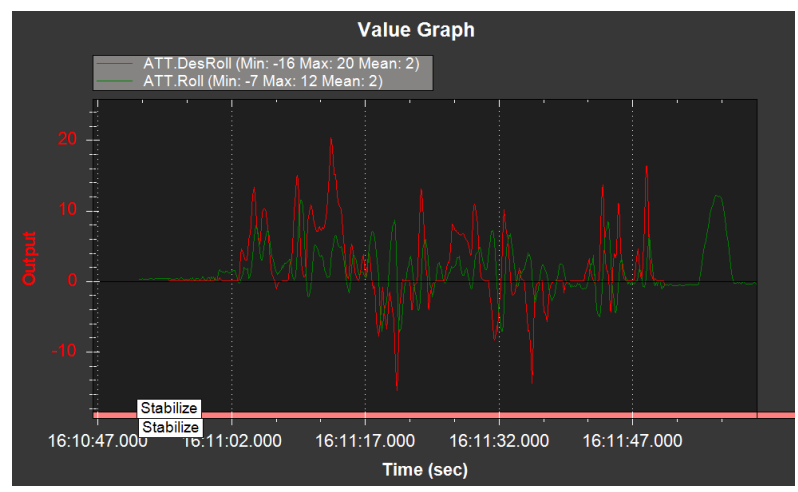

Fig. 15. Graph for desired roll and roll response axis

\section{Conclusion}

Based on the theoretical study and flight data analysis, the proposed hybrid VTOL UAV system would achieve the predicted flight performance in terms of stability and flight range. This study encountered some challenges where the fabrication of the prototype cannot be completed within the specified time due to delay in receiving new part (motors) due to defect and also sourcing the right material for the wing.

\section{Future Work}

The model VTOL-UAV assembly is still in progress. Main components such as the motors/ and RCs are functional well. Below show some of the pictures of parts or and the construction of the assembly which is still in progress.

The design and development of "VTOL" vertical takeoff and landing UAV prototype is really a preliminary stage in the design, and commercialization of this platform needs much more improvement. With the limitation of facilities, budget and expertise, it is difficult to release the prototype to fully meeting the targeted objectives. The VTOL is not only mechanical product that requires Only mechanical expertise, it does require competency in electronic and RF (Radio frequency) communication as well. Using COTF solution for the electronic and communication could be adequate as starting point in the study phase but customization does require in order to be able to meet the desire targets I,e. range, robustness, interference-free etc.

The proposed product is not a hobby kind of UAV, it is rather to be used for the professional application. Certification and validation by authorized agencies such as DCA and SIRIM are required. The following are recommendation and steps in order to proceed the project.

\section{References}

1. A. K. Patra, K. Patel, G. S. Bundela, and V. P, "Design and Development of Transition Autopilot For VTOL UAV," Int. J. Innov. Eng. Res. Technol., vol. 4, no. 6, pp. 57-72 (2017)

2. D. Jo and Y. Kwon, “Analysis of VTOL UAV Propellant Technology,” J. Comput. Commun., vol. 5, pp. 76-82 (2017)

3. V. Kadamatt, “Tricopter vs Quadcopter vs Hexacopter: A Comparison,” Droney Bee, 2017. [Online]. Available: http://www.droneybee.com/tricopter-vs-quadcopter-vs-hexacopter/. [Accessed: 24-Oct-2017].

4. F. Thomas, "What is a Tricopter, Quadcopter, Hexacopter and Octocopter," Droners Guides, 2017 [Online] Available: https://www.dronersguides.com/what-is-a-quadcopter/. [Accessed: 24-Oct-2017].

5. S. Said, "Quadcopter Mechanics," 2014. [Online]. Available: http://uavsociety.blogspot.my/2014/06/quadcopter-mechanics.html. [Accessed: 24-Oct-2017]

6. J. Yoon, “Adverse Yaw and Aircraft Turns," 2001. [Online]. Available: http://www.aerospaceweb.org/question/dynamics/q0045.shtml. [Accessed: 24-Oct-2017]

7. J. Dryden and R. Barbaccia, "Quadcopter Design Project," (2014)

8. L. Zhong, H. Yuqing, Y. Liying, and H. Jianda, "Control techniques of tilt rotor unmanned aerial vehicle systems : A review,” Chinese J. Aeronaut., vol. 30, no. 1, pp. 135-148, (2017)

9. M. F. Saharudin, "Development of tilt-rotor unmanned aerial vehicle ( UAV ): material selection and structural analysis on wing design," in IOP Conference Series: Materials Science and Engineering (2017) 